\title{
Low temperature optimized dyeing of cotton, wool and silk with extract of camellia sinensis (tea leaves)
}

\begin{abstract}
Low temperature dyeing with Camellia sinensis (Tea leaves) extract has been carried out, mainly developed for the sake of energy conservation. The natural dye extract has been used for dyeing natural materials such as wool fibers, cotton and silk fabrics. Many research groups are working in this area of developing better and optimized dyeing process. The drive has been to reduce energy consumption and to make the natural dyeing process ecofriendly by reducing the use of metal mordant. In the present study innovative dyeing has been done at $40^{\circ} \mathrm{C}$ with the use of minimal amounts of metal mordant with tea extract. It has shown good dyeing results. Pretreatment with only $1-2 \%$ metal mordant and $5 \%$ of plant extract (owf) shows very good fastness properties for cotton, wool and silk dyed materials. The role of metal mordant in conjunction with Camellia was found to enhance the dyeability and fastness properties effect in case all three types of material, which otherwise showed poor effects. Thus the net enhancement of dye uptake due to metal mordanting has been found to be $64-67 \%$, in cotton, $70-75 \%$ in silk and $67-72 \%$ in wool with respect to controlled samples. The higher percentage of color strength in the case of silk and wool makes tea leaves best suited for these natural materials.
\end{abstract}

Volume 2 Issue I - 2017

\author{
Padma SVankar,' Dhara Shukla,' Samudrika \\ Wijayapala ${ }^{2}$ \\ 'Facility for Ecological and Analytical Testing, Indian Institute of \\ Technology, India \\ ${ }^{2}$ Department of Textile and Clothing, University of Moratuwa, \\ Sri Lanka
}

\begin{abstract}
Correspondence: Padma S Vankar, Facility for Ecological and Analytical Testing, Indian Institute of Technology, Kanpur-208 016 , India, Email padma.vankar@gmail.com
\end{abstract}

Received: February 17, 2017| Published: June 12, 2017

Keywords: low temperature dyeing, camellia sinensis, natural dye, cotton, wool, silk, metal mordents

Abbreviations: EC, epicatechin; EGC, epicatechin gallate; EGCG, epigallocatechin gallate; AAS, atomic absorption spectrometer

\section{Introduction}

Camellia sinensis or Tea is an evergreen plant. Tea leaves contain many compounds, such as poly phenols (catechins and flavonoids). Tea tannins called Catechins include gallocatechin, epicatechin (EC), epigallocatechin (EGC), epicatechin gallate (EGC) and epigallocatechin gallate (EGCG) has been considered as potential natural dye source. Cotton and jute fabrics were dyed with an aqueous extract of tea, containing tannins as the main colorant species. ${ }^{1}$ Earlier attempts of using tea extract for wool dyeing was carried out with and without metal salts as mordants, using three different dyeing methods: pre-mordanting, meta-mordanting and post-mordanting. They utilized four different kinds of tea leaves. About one third of the catechin was shown to be absorbed by wool fabric during dyeing. ${ }^{2}$ A study was conducted with green tea and black tea with the purpose of assessing the dyeing properties. The extracted dyes were applied with and without mordants on silk fabric. ${ }^{3}$

In another study of tea extract dyeing, elaborate extraction conditions, including solid-liquid ratio, extraction temperature, acidity of extractant and reaction time was shown to have immense effect on the stability of dyes. The dye was significantly affected by $\mathrm{pH}$ value, oxidant and reductant, but its thermal stability was good. Metal irons such as $\mathrm{Ca}^{2+}, \mathrm{Cu}^{2+}, \mathrm{Zn}^{2+}, \mathrm{Mg}^{2+}, \mathrm{Na}^{+}, \mathrm{K}^{+}, \mathrm{Pb}^{2+}, \mathrm{Sn}^{2+}$ and $\mathrm{Ni}^{2+}$ had no effect on the color, but $\mathrm{Al}^{3+}, \mathrm{Fe}^{2+}$ and $\mathrm{Fe}^{3+}$ had harmful effect. ${ }^{4}$

Kaur used green tea for dyeing cotton; the dyeing was carried out by using with and without metal mordants and the light and wash fastnesses were found to be moderate to good. ${ }^{5}$
He et al. ${ }^{6}$ found that leaching tea pigments for dyeing cotton fabrics gave fairly good results and they discussed the influence of the factors of dyeing.

In the present study black tea leaves powder from Srilankan origin have been used as natural dye-stuff used as indigenous source which has been developed scientifically for shades of light brown to dark brown as shown in cotton, silk and wool dyed samples. Use of Tea dust, a waste product of tea industry has been emphasized herein as shown in Figures $1 \& 2$. Thus in this work an optimized process in terms of usage of metal mordants, dyeing time, temperature and dye extract for three natural fabrics with good color fastness properties and dye adherence with Tea Leaves (as dye) has been tried to develop.

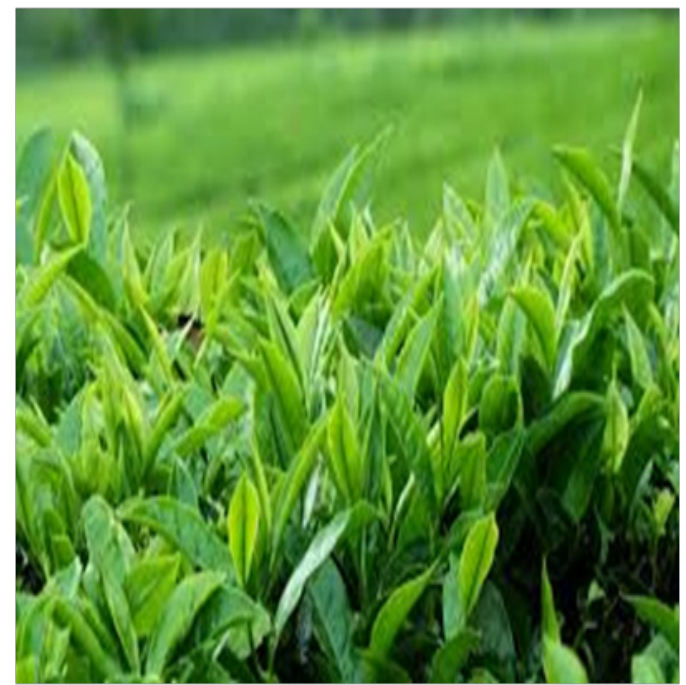

Figure I Srilankan Tea plantation. 


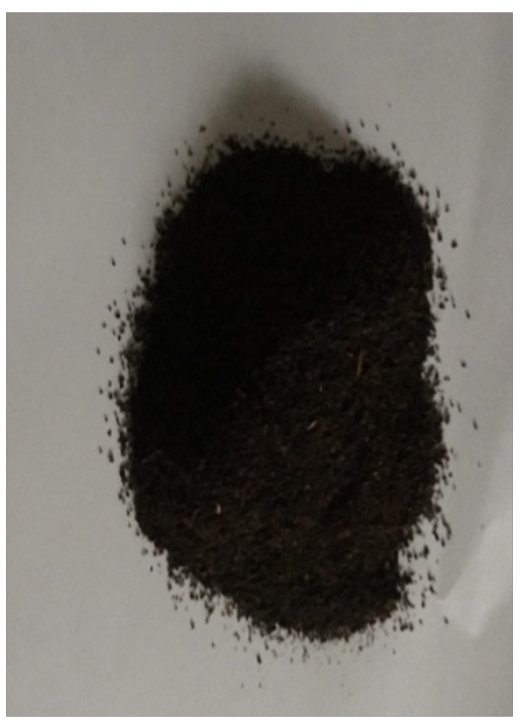

Figure 2 Srilankan Tea dust.

\section{Experimental}

Detailed experimental conditions have been described. More experiments have been added.

\section{Materials}

The cotton fabric of 105 GSM was scoured with a solution containing $5 \mathrm{~g} / 1$ of sodium carbonate and $3 \mathrm{~g} / 1$ of non-ionic detergent (Labolene) under the boiling condition for $4 \mathrm{~h}$, after which time it was thoroughly rinsed and air dried at room temperature.

Munga silk of GSM- 45 fabric and pure new wool 34/S yarn count were scoured with solution containing $0.5 \mathrm{~g} / \mathrm{L}$ sodium carbonate and $2 \mathrm{~g} / \mathrm{L}$ non-ionic detergent (Labolene) solution at $40-45^{\circ} \mathrm{C}$ for $30 \mathrm{~min}$, keeping the material to liquor ratio at 1:50. The scoured material was thoroughly washed with tap water and dried at room temperature. The scoured material was soaked in clean water for $30 \mathrm{~min}$ prior to dyeing or mordanting. Chemicals as Tannic acid, alum, ferrous sulphate, stannic chloride, stannous chloride, copper sulphate and potassium dichromate were procured from SD Fine Chemicals, Kanpur.

\section{Dye material}

Camellia sinensis leaves powder (dust) from Srilankan was used as dye.

\section{Methods}

Extraction of the dye: Dry leaves powder (dust) was heated in distilled water at $\left(90-100^{\circ} \mathrm{C}\right)$ in a beaker kept over water bath for quick extraction of the colorant for 2-3hours, then the filtrate was filtered. The extract was concentrated in rotatory evaporator till the volume became half and then used for dyeing.

Fabric treatment of before dyeing: Scouring of fabric is an important step in cotton dyeing when using natural dyes. The fabric was treated with $4 \%$ (owf) solution of tannic acid prepared in water. ${ }^{6}$ The fabric should be dipped in tannic acid solution for at least 4-5hours and must be covered to avoid patchy stains on the fabric, it is squeezed and dried. Pre-mordanting was used for this study, fabric which was already treated with tannic acid was dipped, in mordant (2\% alum and ferrous sulphate, $1 \%$ each of stannous chloride, stannic chloride, copper sulphate and potassium dichromate separately) solution and was kept on water bath at $40^{\circ} \mathrm{C}$ for one hour. It was squeezed and dried. Silk and wool were directly premordanted with metal salts; no tannic acid treatment was required in the latter cases.

\section{Dyeing}

Two step dyeing process: A two step dyeing (in the ratio of $1-2 \%$ mordant, owf) was used as pretreatment and then dyeing with Tea leaves powder extract was carried out for 3 hour at temperature $40^{\circ} \mathrm{C} .^{7}$

Dye fixing: The dyed fabrics derived from all the above mentioned processes were dipped for $15 \mathrm{~min}$ in dye-fixing solution which consists of sodium chloride solution ( $2 \% \mathrm{w} / \mathrm{v}$ with respect to the fabric) and then rinsed thoroughly in tap water, before leaving to dry in open air.

\section{Metal analysis of dyed fabrics}

Metal analyses of Dyed samples were carried out by Atomic Absorption Spectrometer (Perkin Elmer) for residual metal content on the dyed fabric and yarn. Metal analysis of dyed fabrics was subjected to acid dissolution followed by AAS standard testing protocol.

\section{Measurements and analysis}

Color measurements: The relative color strength of dyed fabrics expressed as K/S was measured by the light reflectance technique using the Kubelka-Munk equation. ${ }^{8,9}$ The reflectance of dyed fabrics was measured on a Premier Color scan.

$$
K / S=(1-R)^{2} / 2 R
$$

Where $\mathrm{R}$ is the decimal fraction of the reflectance of dyed fabric.

\section{Identification of extracted dye}

Spectroscopic and chromatographic analysis of extract: The extracted dye was identified by characterization through various techniques like

i. Ultra violet-visible spectroscopy-through UV-Visible spectrophotometer (Heinous $\alpha$ UV lamda).

ii. Fourier transform-infra-red spectroscopy -was recorded on Vertex 70 model of Bruker. IR sample was prepared by completely dry methanolic extract of Tea leaves.

Fastness testing of dyed samples: Following are the machines used:

a. Xenoster: Used to test the light fastness of the dyed fabric.

b. Wash wheel: Thermo lab model: Used to test the washing fastness of the dyed fabric.

c. Perspirometer: Sashmira Model: Used for the testing of perspiration fastness of the dyed fabric.

d. Crock meter: Used for testing the rubbing fastness of the dyed fabric (Ravindra Eng. Model).

\section{Results and discussions}

There is always a need of newer shades for dyed fabric in dyeing industry and this research provides beautiful and earthy shades of pastel brown which are generally not available among the known 
natural dye shade cards. Innovative dyeing with Camellia sinensis (Tea leaves) has been done at $40^{\circ} \mathrm{C}$ (low temperature) with the use of minimal amounts of metal mordant.

Although Tea leaves contain tannins, but the activation on cotton can only be done by specific tannin variety called tannic acid which solubilizes well in water and coats on cotton for better dye adherence on cotton. The tannins found in tea are called thearubigins, a class of chemicals which includes theaflavins. These chemicals are formed in black tea when the antioxidants inherent in green tea, called catechins, become oxidized. Tea does not contain any tannic acid. Hence for the better dye adherence on cotton tannic acid pretreatment is required and essential. It is a total process of room temperature dyeing only the extract is prepared by boiling. Tannic acid pretreatment, metal mordanting and dyeing are all carried out at room temperature.

\section{UV-Visible spectrum of tea leaves extract}

The methanolic extract from Camellia shows peak at $211 \mathrm{~nm}$ $\left(2.500 \mathrm{~A}^{\circ}\right), 274 \mathrm{~nm}\left(0.922 \mathrm{~A}^{\circ}\right)$ in the $\mathrm{UV}$ region and $470 \mathrm{~nm}\left(1.058 \mathrm{~A}^{\circ}\right)$, $665 \mathrm{~nm}\left(0.410 \mathrm{~A}^{\circ}\right)$ in the Visible region as shown in the Figure 3. These peaks explain the presence of colorant moiety in the visible range and UV range and are very characteristic for identifying chromophores in tea leaves responsible for brown shades on fabric after dyeing.

\section{FT-IR spectrum of the extract}

The dry extract from camellia shows the prominent peaks in the area of 3336, 2922, 2852, 1700, 1657, 1428, 1093, 1020, 745, 671, 609 $\mathrm{cm}^{-1}$ as shown in Figure 4. The intense peak at $3336 \mathrm{~cm}^{-1}$ verifies the presence of poly phenolic groups which are abundantly present in this plant. These poly phenols help in interlocking majority chromophore to get adhere on fabric and thus increase dye fastness. This spectrum is very characteristic for tea leaves and thus shows the presence of all the functional groups responsible for color in tea extract.

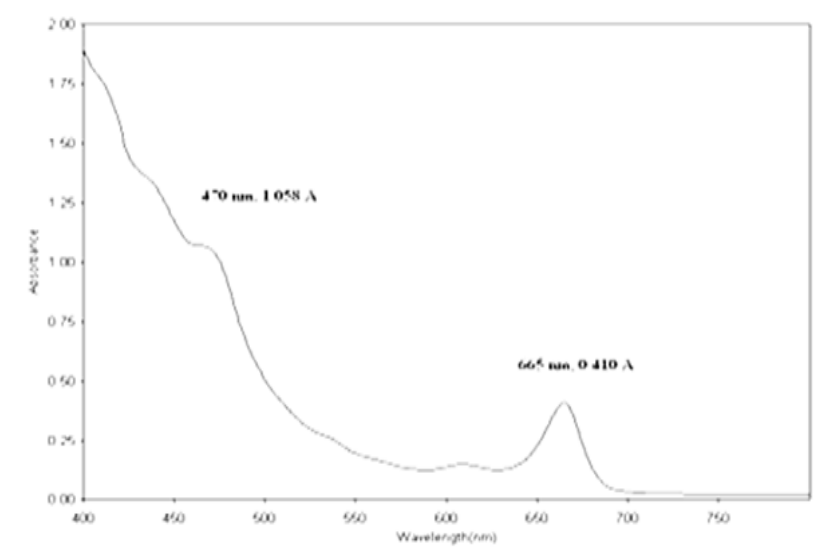

Figure 3 UV-Visible spectrum of Camellia.

\section{Dyeing with tea leaves extract}

Dyeing was carried out in two steps i.e. pretreatment with metal mordant and then dyeing with Tea leaves powder extract for 3 hour at temperature $40^{\circ} \mathrm{C}$. Then dyed fabrics were fixed with Sodium Chloride (brine) solution. The role of brine acting as dye fix may be understood as the following- it acts like an electrolyte for migration, adsorption and fixation of the dyestuff to the cellulose and other fiber materials.

The colorimetric data obtained from dyed fabrics and yarn which had been pretreated with tannic acid/metal mordants in the case of cotton and only metal mordants in the cases of silk and wool reveal that pretreatment markedly improved the wash fastness, in terms of change of shade of the dyed fabrics with respect to controlled samples. It also increased the color strength and flattened the shade of the dyeing. In each experiment controlled dyed samples were also prepared. The novelty of this research is room temperature dyeing, an energy saving process very appropriate for industrial scale up.

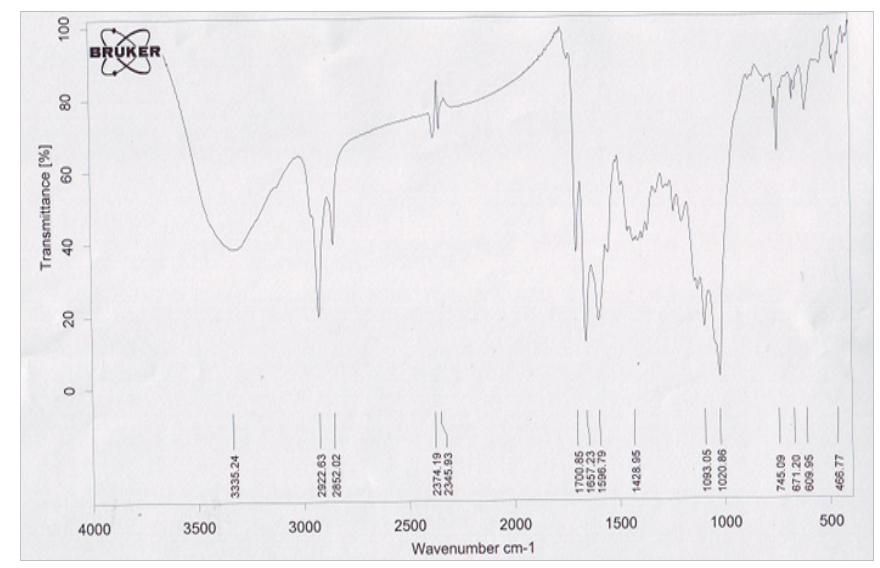

Figure 4 FT-IR spectrum of Camellia.

\section{Color measurements}

In the case of natural dyes the strength is calculated at each wavelength and average is taken as real strength of the colorant as the colorant is not a single molecule most of time. This is becoming more and more popular. Sometimes, it may not give correct strength idea of the colorant as reflectance values are changing wavelength to wavelength. Strength at R Min is the correct representative but strength based on integrated wavelength is preferred by most of the users. In case of mixture dyes, it is found very useful. This is useful if the dye is a mixture dye, thus there was a need of recording changes in $\mathrm{K} / \mathrm{S}$ values with respect to different wavelengths. K/S was measured for dyed cotton, silk fabrics and wool yarn as shown in Figures 5-7.

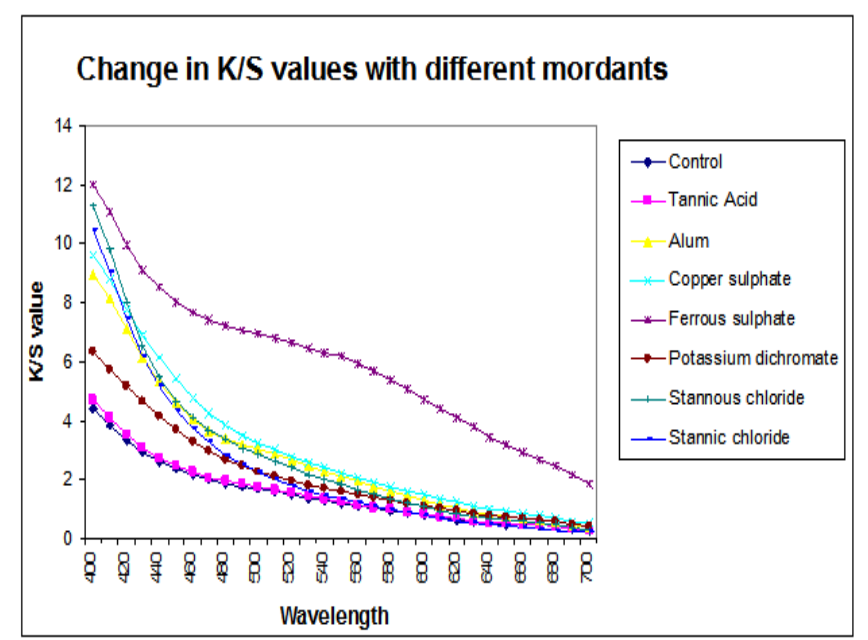

Figure 5 Change in $\mathrm{K} / \mathrm{S}$ values with different mordants for cotton fabric.

\section{Effect of mordanting conditions}

It was observed that the pre mordanting technique with metal mordants imparted good fastness properties to the cotton, wool and silk fabric due to formation of complex as mentioned for alum. ${ }^{10}$ 
Control samples without mordant were also prepared for comparison. Therefore, in premordanting technique, the dyed fabrics were mordanted with $1 \%$ of each stannic chloride, stannous chloride, copper sulphate, potassium dichromate and $2 \%$ of ferrous sulphate and alum. The excessively high $\mathrm{K} / \mathrm{S}$ value for cotton sample with ferrous mordant is due to the fact that a water soluble ferrous tannate complex is formed. Because of its solubility, the complex is able to penetrate the fabric surface in presence of air a ferric tannate pigment is formed. This new complex is not water-soluble, contributing to its enhancement in washing fastness. Similar results of the K/S values for silk and wool were observed.

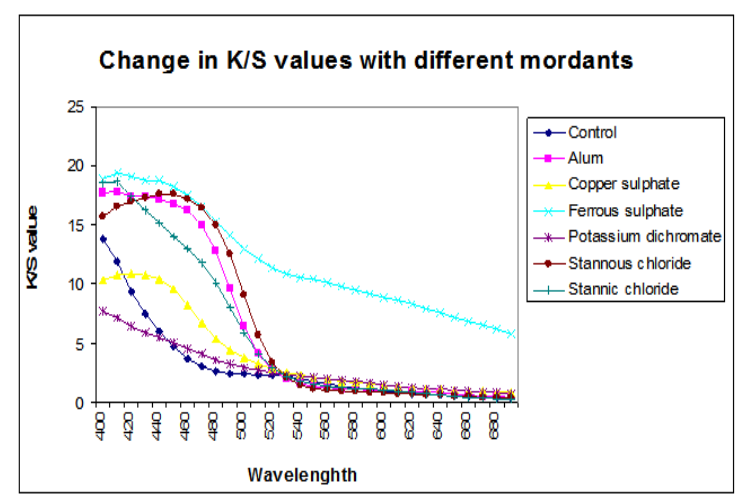

Figure 6 Change in K/S values with different mordants for silk fabric.

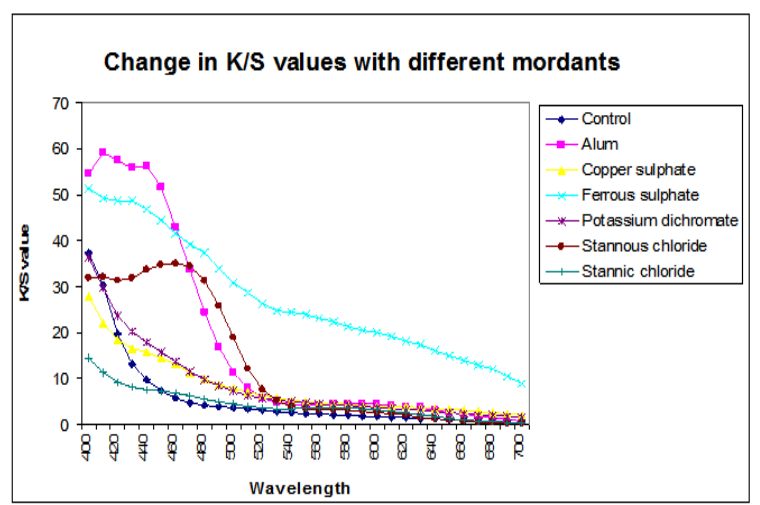

Figure 7 Change in K/S values with different mordants for wool yarn.
The mordant activity of the six cases followed the sequences. Tables 1a-1c shows the colorimetric results of the dyed samples.

The order of $\mathrm{K} / \mathrm{S}$ values is as following: $\mathrm{Fe} \rightarrow \mathrm{Cu} \rightarrow \mathrm{Al} \rightarrow \mathrm{Sn}$ (IV) $\rightarrow$ Sn (II) $\rightarrow$ Cr in cotton for Camellia, the absorption of color by cotton fabric was enhanced when using metal mordants. In silk the $\mathrm{K} / \mathrm{S}$ values showed $\mathrm{Fe} \rightarrow \mathrm{Cu} \rightarrow \mathrm{Al} \rightarrow \mathrm{Cr} \rightarrow \mathrm{Sn}(\mathrm{IV}) \rightarrow \mathrm{Sn}$ (II) order, the absorption of color by silk fabric was enhanced when using metal mordants particularly metal mordants $\mathrm{Fe}(\mathrm{II})$ provides best chelation in all the cases due to empty d-orbitals. The same order was also observed for wool due to similar reasons. It is proposed ${ }^{11}$ that low water solubility, large molecular size, iron tannate complex was formed that occupied peripheral regions of the dyed fibre attributing dye adherence.

\section{Fastness testing}

The dyed samples were tested according to Indian standard methods. ${ }^{12}$ The specific tests were: color fastness for light, IS-245485 by xenotester, color fastness to rubbing, IS-766- 88 by crock meter, color fastness to washing; and IS-687-79 by wash wheel, color fastness to perspiration, IS-971-83 by perspirometer. Results are shown in table-5 of metal mordanted samples for the two step dyeing conditions.

Thus Table 2 shows that alum, ferrous sulphate and copper sulphate mordanting has caused improved fastness properties with all the three types of material. Marked improvement can be noticed in the case of washing and light fastness. Thus the dye can be strongly recommended for commercial use as the source of the dye is very cheap and readily available. Shades of brown ranging from camel color to chocolate brown can be easily obtained by tea leaves powder aqueous extract. Table 3 shows the shade card for all the samples dyed by Camellia.

\section{Metal analysis}

Analysis of metal residues were also evaluated by Atomic Absorption Spectrometer (AAS) and found to have $<0.05 \mathrm{ppm}$ in all cases of dyed fabrics. This makes it safe for using copper sulphate and potassium dichromate mordants which are otherwise not recommended in ecofreindly natural dyeing process. The residual metal content in the dye bath was $<0.50 \mathrm{ppm}$ in all the cases. Thus making the process ecofreindly and safe.

Table I a CIE Lab Values of Tea leaves dyed Cotton samples

\begin{tabular}{lllllllll}
\hline Parameters & Control & Tannin & Alum & $\mathrm{CuSO}_{4}$ & $\mathrm{FeSO}_{4}$ & $\mathrm{~K}_{2} \mathrm{Cr}_{2} \mathrm{O}_{7}$ & $\mathrm{SnCl}_{4}$ & $\mathrm{SnCl}_{2}$ \\
\hline $\mathrm{L}^{*}$ & 57.2 & 57.23 & 58.404 & 58.764 & 53.756 & 58.148 & 60.126 & 62.289 \\
$\mathrm{a}^{*}$ & 7.753 & 8.089 & 8.95 & 6.822 & 5.288 & 6.134 & 9.145 & 8.795 \\
$\mathrm{~b}^{*}$ & 19.166 & 19.242 & 21.888 & 22.986 & 8.832 & 21.509 & 25.7 & 30.476 \\
$\mathrm{C}^{*}$ & 20.675 & 20.873 & 23.647 & 23.977 & 10.294 & 22.367 & 27.279 & 31.72 \\
$\mathrm{H}^{*}$ & 67.948 & 67.172 & 67.733 & 73.053 & 59.066 & 74.053 & 70.384 & 73.873 \\
$\mathrm{~K} / \mathrm{S}$ & 27.912 & 29.081 & 52.818 & 59.58 & 114.595 & 41.365 & 52.359 & 45.163 \\
$\mathrm{dE}$ & - & 0.346 & 3.208 & 4.231 & 11.168 & 3.002 & 7.293 & 12.446 \\
\hline
\end{tabular}


Table I b CIE Lab Values of Tea leaves dyed Silk samples

\begin{tabular}{llllllll}
\hline Parameters & Control & Alum & $\mathrm{CuSO}_{4}$ & $\mathrm{FeSO}_{4}$ & $\mathrm{~K}_{2} \mathrm{Cr}_{2} \mathrm{O}_{7}$ & $\mathrm{SnCl}_{4}$ & $\mathrm{SnCl}_{2}$ \\
\hline $\mathrm{L}^{*}$ & 58.82 & 59.978 & 59.609 & 52.908 & 58.924 & 60.399 & 59.634 \\
$\mathrm{a}^{*}$ & 5.686 & 6.68 & 5.83 & 3.719 & 4.05 & 6.591 & 4.34 \\
$\mathrm{~b}^{*}$ & 21.607 & 23.904 & 23.622 & 3.549 & 21.718 & 24.801 & 22.056 \\
$\mathrm{C}^{*}$ & 22.343 & 24.84 & 24.331 & 5.141 & 22.092 & 25.662 & 22.479 \\
$\mathrm{H}^{*}$ & 75.226 & 74.357 & 76.106 & 43.643 & 79.405 & 75.087 & 78.836 \\
$\mathrm{~K} / \mathrm{S}$ & 26.979 & 34.692 & 45.397 & 248.837 & 34.623 & 34.548 & 25.672 \\
$\mathrm{dE}$ & - & 2.758 & 2.169 & 19.103 & 1.643 & 3.676 & 1.636 \\
\hline
\end{tabular}

Table Ic CIE Lab Values of Tea leaves dyed Wool samples

\begin{tabular}{llllllll}
\hline Parameters & Control & Alum & $\mathrm{CuSO}_{4}$ & $\mathrm{FeSO}_{4}$ & $\mathrm{~K}_{2} \mathrm{Cr}_{2} \mathbf{O}_{7}$ & $\mathrm{SnCl}_{4}$ & $\mathrm{SnCl}_{2}$ \\
\hline $\mathrm{L}^{*}$ & 51.197 & 50.642 & 49.482 & 43.537 & 50.633 & 52.79 & 52.788 \\
$\mathrm{a}^{*}$ & $11.84 \mathrm{I}$ & 10.617 & 6.285 & 3.617 & 5.633 & 13.333 & 13.269 \\
$\mathrm{~b}^{*}$ & 24.455 & 22.884 & 20.347 & 1.748 & 23.093 & 28.073 & 28.063 \\
$\mathrm{C}^{*}$ & $27.17 \mathrm{I}$ & 25.227 & 21.296 & 4.017 & 23.77 & 31.078 & 31.042 \\
$\mathrm{H}^{*}$ & 64.138 & 65.085 & 72.805 & 25.783 & 76.261 & 64.569 & 64.668 \\
$\mathrm{~K} / \mathrm{S}$ & 48.94 & 31.75 & 112.882 & 252.675 & 30.654 & 28.475 & 28.47 \\
$\mathrm{dE}$ & - & 2.067 & 7.119 & 25.336 & 6.381 & 4.225 & 4.194 \\
\hline
\end{tabular}

Table 2 Fastness properties of dyed cotton, silk fabrics and wool yarn with Camellia

\begin{tabular}{|c|c|c|c|c|c|c|}
\hline \multirow[t]{2}{*}{ Dyeing methods } & \multicolumn{6}{|c|}{ Wash-perspiration-rubbing-light } \\
\hline & WF & Per acidic & Per basic & Rub dry & Rub wet & LF \\
\hline Cotton (control) & 4-Mar & 3 & 3 & 3 & 3 & 4-Mar \\
\hline Cotton(Alum) & 4 & 4 & 4-Mar & 4-Mar & 4-Mar & 4 \\
\hline Cotton $\left(\mathrm{Fe} \mathrm{SO}_{4}\right)$ & 5-Apr & 4 & 4 & 4 & 4 & 5-Apr \\
\hline Cotton( $\left.\mathrm{Cu} \mathrm{SO}_{4}\right)$ & 4 & 4 & 4 & 4 & 4 & 4 \\
\hline Wool (control) & 3 & 3 & 3 & 3 & 3 & 3 \\
\hline Wool(Alum) & 4 & 4 & 4 & 4 & 4 & 4 \\
\hline Wool $\left(\mathrm{Fe} \mathrm{SO}_{4}\right)$ & 5 & 5-Apr & 5-Apr & 5-Apr & 5-Apr & 5 \\
\hline Wool $\left(\mathrm{Cu} \mathrm{SO}_{4}\right)$ & 5-Apr & 4 & 4 & 4 & 4 & 5-Apr \\
\hline Silk (control) & 3 & 3 & 3 & 3 & 3 & 3 \\
\hline Silk(Alum) & 4 & 4 & 4 & 4 & 4 & 4 \\
\hline Silk( $\left.\mathrm{Fe} \mathrm{SO}_{4}\right)$ & 5 & 5-Apr & 5-Apr & $5-A p r$ & $5-A p r$ & 5-Apr \\
\hline Silk( $\left.\mathrm{Cu} \mathrm{SO}_{4}\right)$ & 5-Apr & 4 & 4 & 4 & 4 & 5-Apr \\
\hline
\end{tabular}


Table 3 Shade card of dyed samples of Camellia

\begin{tabular}{|c|c|c|c|}
\hline Mordant & Cotton & Silk & Wool \\
\hline \multicolumn{4}{|l|}{ Control } \\
\hline \multicolumn{4}{|l|}{ Alum } \\
\hline \multicolumn{4}{|l|}{ Cu Sulphate } \\
\hline \multicolumn{4}{|l|}{ Fe Sulphate } \\
\hline \multicolumn{4}{|l|}{ Pot. Dichrom. } \\
\hline \multicolumn{4}{|l|}{ Sn Chloride(II) } \\
\hline Sn Chloride(IV) & & & \\
\hline
\end{tabular}

\section{Conclusion}

Industrial crop tea and its powder waste have been used as dye source. Camellia sinensis has been shown to have good dyeing prospects through this study. It grows abundantly in India and Srilanka and can be explored for commercial natural dyeing particularly the waste that is generated in the Tea leaves processing. $1-2 \%$ of metal mordant in conjunction with Camellia was found to enhance the dyeability and fastness properties effect in case all three types of material. Highlights of this process are low temperature dyeing $\left(40^{\circ} \mathrm{C}\right)$, use of low metal mordant percentage and dye effluents are less contaminated with metal salts, which is a advantageous for industrial natural dyeing, being energy saving and ecofriendly. Thus the net enhancement of dye uptake due to metal mordanting has been found to be $64-67 \%$ in cotton, $70-75 \%$ in silk and $67-72 \%$ in wool with respect to control samples. The higher percentage of color strength in the case of silk and wool makes tea leaves best suited for these natural materials. However when attempts were made with polyester and nylon fabric the dyeing results was not good. Thus tea leaves dust powder is ideally suited for natural fibres which includes bamboo fabric. ${ }^{13}$ As compared to the conventional dyeing method room temperature dyeing is a very energy saving process.

\section{Summary}

Innovative dyeing with Camellia sinensis (Tea leaves) has been done at $40^{\circ} \mathrm{C}$ (low temperature) with the use of minimal amounts of metal mordant with tea extract. Pretreatment with only $1-2 \%$ metal mordant and tea extract (owf) shows very good fastness properties for cotton, wool and silk dyed fabrics. It has shown good dyeing results.

\section{Acknowledgments}

None.

\section{Conflict of interest}

Author declares there is no conflict of interest in publishing the article.

\section{References}

1. Deo HT, Desai BK. Dyeing of cotton and jute with tea as a natural dye. Coloration Technology. 2006;115(7-8):224-227.

2. Chan PM, Yuen CWM, Yeung KW. Dyeing- natural-dye wool-fiber. Textile Asia. 2000;31(2):28-30.

3. Kim S. The application of tea dyeing to silk. Journal of Dong Hua University. 2001;18(3):58-61

4. Wu R, Lai Y, Ou S, et al. Extraction of Natural Tea Dyes and Its Application on Silk Fabrics. J Advanced Textile Technology. 2011;5(19):1-4.

5. Kaur V. Dyeing of Cotton with Tea as Natural dye. International J Eng Innovation \& Res. 2015;4(1):184-187.

6. He Z, Wu Z. The Application Research on Cotton Fabric of Natural Tea Pigments. Advances in Intelligent \& Soft Computing. 2012;158:365-372.

7. Vankar P. Dyeing with tectona leaves dye. Asian Textile $J$. 2003;12(4):86-88.

8. Kubelka P. New Contributions to the Optics of Intensely Light-Scattering Materials. Part I. J Opt Soc Am. 1948;38(5):448-457.

9. Kubelka P. New Contributions to the Optics of Intensely Light-Scattering Materials. Part II: Non homogeneous Layers. J Opt Soc Am. 1954;44(4):330-335.

10. Septhum C, Rattanaphani V, Rattanaphani S. UV-Vis spectroscopic study of natural dyes with alum as a mordant. Suranaree J Science Technol. 2007;14(1):91-97. 
11. Burkinshaw SM, Kumar NA. A tannic acid/ferrous sulfate after treatment for dyed nylon 6, 6. Dyes \& Pigments. 2008;79(1):48-53.

12. Indian Standards Institution B. Handbook of Textile Testing. New Delhi, India; 2000.
13. Kaur S, Chattopadhyay DP, Kaur V. Dyeing of Bamboo with Tea as Natural dye. Res J Eng Sci. 2012;1(4):21-26. 